\title{
A!
}

This is an electronic reprint of the original article.

This reprint may differ from the original in pagination and typographic detail.

Kinnunen, Jami J.; Wu, Zhigang; Bruun, Georg M.

\section{Induced p -Wave Pairing in Bose-Fermi Mixtures}

Published in:

Physical Review Letters

DOI:

10.1103/PhysRevLett.121.253402

Published: 19/12/2018

Document Version

Publisher's PDF, also known as Version of record

Please cite the original version:

Kinnunen, J. J., Wu, Z., \& Bruun, G. M. (2018). Induced p -Wave Pairing in Bose-Fermi Mixtures. Physical Review Letters, 121(25), 1-6. [253402]. https://doi.org/10.1103/PhysRevLett.121.253402

This material is protected by copyright and other intellectual property rights, and duplication or sale of all or part of any of the repository collections is not permitted, except that material may be duplicated by you for your research use or educational purposes in electronic or print form. You must obtain permission for any other use. Electronic or print copies may not be offered, whether for sale or otherwise to anyone who is not an authorised user. 


\title{
Induced $p$-Wave Pairing in Bose-Fermi Mixtures
}

\author{
Jami J. Kinnunen* \\ Department of Applied Physics, Aalto University, FI-00076 Aalto, Finland \\ Zhigang $\mathrm{Wu}^{\dagger}$ \\ Shenzhen Institute for Quantum Science and Engineering and Department of Physics, \\ Southern University of Science and Technology, Shenzhen 518055, China \\ Georg M. Bruun \\ Department of Physics and Astronomy, Aarhus University, DK-8000 Aarhus C, Denmark
}

(Received 13 September 2018; published 19 December 2018)

\begin{abstract}
Cooper pairing caused by an induced interaction represents a paradigm in our description of fermionic superfluidity. Here, we present a strong coupling theory for the critical temperature of $p$-wave pairing between spin polarized fermions immersed in a Bose-Einstein condensate. The fermions interact via the exchange of phonons in the condensate, and our self-consistent theory takes into account the full frequency and momentum dependence of the resulting induced interaction. We demonstrate that both retardation and self-energy effects are important for obtaining a reliable value of the critical temperature. Focusing on experimentally relevant systems, we perform a systematic analysis varying the boson-boson and bosonfermion interaction strength as well as their masses, and identify the most suitable system for realizing a $p$-wave superfluid. Our results show that such a superfluid indeed is experimentally within reach using light bosons mixed with heavy fermions.
\end{abstract}

DOI: $10.1103 /$ PhysRevLett.121.253402

The theory of Cooper pairing of electrons due to an induced attractive interaction mediated via crystal phonons successfully explained the origin of superconductivity and stands out as a highlight of quantum many-body physics [1]. Interest in pairing with non- $s$-wave symmetry began with the understanding of superfluidity in ${ }^{3} \mathrm{He}$ [2] and has increased further with the advent of systems such as the copper and iron based high temperature superconductors $[3,4]$, for which many fundamental questions remain. Ultracold atoms have emerged as a powerful platform to explore such many-body physics, and the realization of strong $s$-wave pairing in a Fermi gas was a landmark achievement $[5,6]$. Pairing in these gases is however brought by a direct attractive interaction between the fermions, and so far no one has realized pairing via an induced interaction in cold atom systems.

Spin-polarized fermions mixed with a Bose-Einstein condensate (BEC) represents a promising setup for realizing a $p$-wave superfluid caused by an induced interaction. Here, the fermions gain an effective attraction through exchanging phonons in the BEC [7,8]. A very attractive feature of such a mediated $p$-wave interaction is that both its strength and range can be tuned by changing the properties of the BEC. Experimentally, much progress has been made recently on atomic mixtures and the list of trapped Bose-Fermi mixtures is already long [9-20]. Cooper pairing in atomic Bose-Fermi mixtures was originally predicted using weak coupling BCS theory [21], and since then several authors have considered the problem using theories with varying degree of sophistication [22-26]. It has furthermore been predicted that topological $p$-wave superfluids can be realized in mixed dimensional Bose-Fermi mixtures [27,28]. However, a strong coupling theory for the critical temperature of a three-dimensional $p$-wave superfluid including the full energy and momentum dependent pairing and self-energy effects in a consistent way, is still lacking.

We present here such a strong coupling theory for the critical temperature $T_{\mathrm{c}}$ of $p$-wave pairing of spin polarized fermions in a BEC. Including the full frequency and momentum dependence of the induced interaction between the fermions caused by the exchange of phonons in the $\mathrm{BEC}$, we show that retardation as well as self-energy effects can significantly suppress $T_{\mathrm{c}}$. We perform a systematic analysis varying both the boson-boson and boson-fermion interaction strengths as well as their mass ratio, with an emphasis on experimentally relevant atomic mixtures. This allows us to determine the most suitable systems and the optimal conditions for which $p$-wave superfluidity due to an induced interaction can be realized.

Model.-We consider a three-dimensional system consisting of spin-polarized, noninteracting fermions of mass $m_{\mathrm{F}}$ and density $n_{\mathrm{F}}$, mixed with bosons of mass $m_{\mathrm{B}}$ and density $n_{\mathrm{B}}$. The Bose gas is weakly interacting so that it can 
be described by Bogoliubov theory well below the critical temperature for Bose-Einstein condensation. At temperature $T=\beta^{-1}\left(\hbar=k_{\mathrm{B}}=1\right)$, the properties of the mixture are described by the partition function

$$
\mathcal{Z}=\int \mathcal{D}(\bar{a}, a) \int \mathcal{D}\left(\gamma^{*}, \gamma\right) e^{-\left(S_{\mathrm{F}}^{0}+S_{\mathrm{B}}+S_{\text {int }}\right)},
$$

where $(a, \bar{a})$ and $\left(\gamma, \gamma^{*}\right)$ are Grassmann and complex fields for the fermions and Bogoliubov phonons, respectively. The action for the free fermions is

$$
S_{\mathrm{F}}^{0}=\sum_{\mathbf{p}, n} \bar{a}(p)\left(-i \omega_{n}+\xi_{\mathbf{p}}\right) a(p),
$$

where $p \equiv\left(\mathbf{p}, i \omega_{n}\right), \omega_{n}=(2 n+1) \pi T$ is a Fermi Matsubara frequency, and $\xi_{\mathbf{p}}=\mathbf{p}^{2} / 2 m_{\mathrm{F}}-\mu_{\mathrm{F}}$ is the free fermion dispersion measured from the chemical potential $\mu_{\mathrm{F}}$ of the Fermi gas. The action for the Bose gas is given by

$$
S_{\mathrm{B}}=\sum_{\mathbf{q} \neq 0, \nu} \gamma^{*}(q)\left(-i \omega_{\nu}+E_{\mathbf{q}}\right) \gamma(q),
$$

where $q \equiv\left(\mathbf{q}, i \omega_{\nu}\right), \omega_{\nu}=2 \nu \pi T$ is a Bose Matsubara frequency, and $E_{\mathbf{q}}=\sqrt{\varepsilon_{\mathbf{q}}\left(\varepsilon_{\mathbf{q}}+2 g_{\mathrm{B}} n_{\mathrm{B}}\right)}$ is the Bogoliubov spectrum. Here $\varepsilon_{\mathbf{q}}=\mathbf{q}^{2} / 2 m_{\mathrm{B}}$ and $g_{\mathrm{B}}=4 \pi a_{\mathrm{B}} / m_{\mathrm{B}}$, where $a_{\mathrm{B}}$ is the boson scattering length. Finally the fermion-boson interaction is

$$
S_{\text {int }}=g \sqrt{\frac{n_{\mathrm{B}}}{\beta \mathcal{V}}} \sum_{\mathbf{q} \neq 0, \nu} \sqrt{\frac{\varepsilon_{\mathbf{q}}}{E_{\mathbf{q}}}}\left[\gamma^{*}(q)+\gamma(-q)\right] \rho(q),
$$

where $\mathcal{V}$ is the system volume, $\rho\left(\mathbf{q}, i \omega_{\nu}\right) \equiv \sum_{\mathbf{p}^{\prime}, n} \bar{a}\left(\mathbf{p}^{\prime}-\mathbf{q}\right.$, $\left.i \omega_{n}-i \omega_{\nu}\right) a\left(\mathbf{p}^{\prime}, i \omega_{n}\right)$, and $g=2 \pi a_{\mathrm{FB}} / m_{\mathrm{r}}$ is the bosonfermion interaction. Here $m_{\mathrm{r}}=m_{\mathrm{F}} m_{\mathrm{B}} /\left(m_{\mathrm{F}}+m_{\mathrm{B}}\right)$ is the reduced mass and $a_{\mathrm{FB}}$ is the fermion-boson scattering length. In Eq. (4) we did not include terms describing the scattering between fermions and uncondensed bosons. Such terms can be neglected for the relatively weak boson-fermion interactions considered here [29,30], i.e., $k_{\mathrm{F}}\left|a_{\mathrm{FB}}\right| \lesssim 1$.

The Bogoliubov fields in Eq. (1) can be integrated out, yielding an effective action for the fermions [7,8,31]

$$
S_{\mathrm{F}}(\bar{a}, a)=S_{\mathrm{F}}^{0}(\bar{a}, a)+\frac{1}{2 \beta \mathcal{V}} \sum_{\mathbf{q}, \nu} V_{\text {ind }}(q) \bar{\rho}(q) \rho(q),
$$

where $V_{\text {ind }}$ is the phonon-mediated interaction given by

$$
V_{\text {ind }}\left(\mathbf{q}, i \omega_{\nu}\right)=g^{2} \frac{n_{\mathrm{B}}}{m_{\mathrm{B}}} \frac{\mathbf{q}^{2}}{\left(i \omega_{\nu}\right)^{2}-E_{\mathbf{q}}^{2}} .
$$

This interaction corresponds to the exchange of one Bogoliubov mode between the fermions, treating the boson-fermion scattering as energy independent, which is valid for $k_{\mathrm{F}}\left|a_{\mathrm{FB}}\right| \lesssim 1$. In the static case $\omega_{\nu}=0$, Eq. (6) is the Fourier transform of the well-known Yukawa interaction with a range given by the BEC coherence length $\xi_{\mathrm{B}}=1 / \sqrt{8 \pi n_{\mathrm{B}} a_{\mathrm{B}}}$.

Eliashberg theory.-In order to describe pairing between fermions due to the mediated interaction [Eq. (6)] with a high critical temperature $T_{\mathrm{c}}$, we use the Eliashberg theory that retains the full energy-momentum dependence of the pairing field and the diagonal self-energy. This framework has proven accurate for strong coupling electronic superconductors where pairing is mediated by phonons [32], which is quite similar to the case at hand.

The Eliashberg theory determines the normal and anomalous Green's functions, defined as $G(p) \equiv-\langle a(p) \bar{a}(p)\rangle$, $F(p) \equiv-\langle a(p) a(-p)\rangle, \quad$ and $\quad F^{\dagger}(p) \equiv-\langle\bar{a}(-p) \bar{a}(p)\rangle$, where the expectation values are time ordered. The Green's functions obey a generalized Dyson equation shown diagrammatically in Fig. 1, which is solved by

$$
G(p)=\frac{i \omega_{n}+\xi_{\mathbf{p}}+\Sigma(-p)}{\left[i \omega_{n}-A(p)\right]^{2}-\left[\xi_{\mathbf{p}}+S(p)\right]^{2}-|\Delta(p)|^{2}}
$$

and

$$
F(p)=\frac{\Delta(p)}{\left[i \omega_{n}-A(p)\right]^{2}-\left[\xi_{\mathbf{p}}+S(p)\right]^{2}-|\Delta(p)|^{2}},
$$

with $F^{\dagger}\left(\mathbf{p}, i \omega_{n}\right)=F\left(\mathbf{p},-i \omega_{n}\right)^{*}$. Here $\Sigma(p)$ is the normal self-energy, where $S(p)=[\Sigma(p)+\Sigma(-p)] / 2$ and $A(p)=$ $[\Sigma(p)-\Sigma(-p)] / 2$ are its real and imaginary parts, and $\Delta(p)$ is the anomalous self-energy. The latter is essentially a momentum and frequency dependent pairing gap. The selfenergies are evaluated using a generalized Hartree-Fock approximation illustrated in Fig. 1, where the Hartree term is absorbed into a redefinition of the chemical potential $\mu_{\mathrm{F}}$. This gives

$$
\Sigma(p)=-\frac{1}{\beta \mathcal{V}} \sum_{p^{\prime}} V_{\text {ind }}\left(p-p^{\prime}\right) G\left(p^{\prime}\right)
$$

for the normal Fock self-energy and

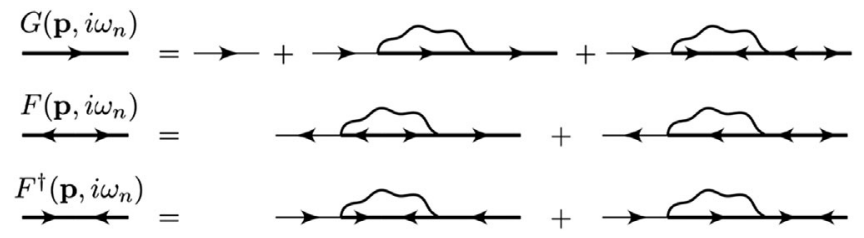

FIG. 1. Diagrammatic structure of the Eliashberg theory. The thin line represents the noninteracting Green's function $G_{0}\left(\mathbf{p}, i \omega_{n}\right)=1 /\left(i \omega_{n}-\xi_{\mathbf{p}}\right)$ and the wavy curve represents the mediated interaction $V_{\text {ind }}\left(\mathbf{q}, i \omega_{\nu}\right)$. 


$$
\Delta(p)=-\frac{1}{\beta \mathcal{V}} \sum_{p^{\prime}} V_{\text {ind }}\left(p-p^{\prime}\right) F\left(p^{\prime}\right)
$$

for the anomalous Fock self-energy. We solve these equations self-consistently for fixed fermion density

$$
n_{\mathrm{F}}=\frac{1}{\beta \mathcal{V}} \sum_{\mathbf{p}, n} G\left(\mathbf{p}, i \omega_{n}\right) e^{i \omega_{n} 0^{+}}
$$

A derivation of the Eliashberg equations using the path integral is given in the Supplemental Material [33].

The pairing gap must be odd in momentum due to the Pauli principle for identical fermions, and it can therefore be expanded in spherical harmonics $Y_{l m}(\hat{\mathbf{p}})$ with $l=1,3, \ldots$ Since $T_{\mathrm{c}}$ is determined from the linearized forms of Eqs. (9)-(11), which do not couple different $(l, m)$ channels, we use the $(l, m)=(1,1)$ ( $p$-wave) ansatz $\Delta\left(\mathbf{p}, i \omega_{n}\right)=\Delta_{11}\left(|\mathbf{p}|, i \omega_{n}\right) Y_{11}(\hat{\mathbf{p}})$, as this yields the highest $T_{\mathrm{c}}$. The normal self-energy $\Sigma(p)$ is spherically symmetric at $T_{\mathrm{c}}$ where there is no pairing to break this symmetry, and so we can write $\Sigma\left(\mathbf{p}, i \omega_{n}\right)=\Sigma_{00}\left(|\mathbf{p}|, i \omega_{n}\right) Y_{00}(\hat{\mathbf{p}})$.

In practice, we determine $T_{\mathrm{c}}$ by first evaluating the normal self-energy self-consistently assuming no pairing. Then we iterate Eqs. (9)-(11) with a finite but very small initial value of the gap function. A decreasing (increasing) gap function under iteration indicates that the given temperature is above (below) $T_{\mathrm{c}}$. The details of the numerical procedure are given in the Supplemental Material [33].

Note that we neglect the effects of the fermions on the bosons and assume a temperature well below the critical temperature of the BEC, so that it can be treated using $T=0$ Bogoliubov theory. This is accurate if the boson density is much larger than that of the fermions, which is often the case experimentally. The effects of a Fermi gas on a BEC were considered in Ref. [34].

Qualitative analysis.-There are four physical parameters that can be independently controlled in this system, namely the Fermi-Bose mass ratio $\alpha \equiv m_{\mathrm{F}} / m_{\mathrm{B}}$, density ratio $n_{\mathrm{B}} / n_{\mathrm{F}}$, scattering length $a_{\mathrm{BF}}$, and the boson scattering length $a_{\mathrm{B}}$. The critical temperature $T_{\mathrm{c}}$ is determined by three dimensionless quantities formed out of these four parameters. The first two are the strength and the range of the mediated interaction, which can be estimated by considering its zero frequency component

$$
V_{\text {ind }}(\mathbf{q}, 0)=-\lambda \frac{\epsilon_{\mathrm{F}} / k_{\mathrm{F}}^{3}}{\left(\mathbf{q} / k_{\mathrm{F}}\right)^{2}+2 /\left(k_{\mathrm{F}} \xi_{\mathrm{B}}\right)^{2}} .
$$

Here, $\epsilon_{\mathrm{F}}=k_{\mathrm{F}}^{2} / 2 m_{\mathrm{F}}$ is the Fermi energy of the system with $k_{\mathrm{F}}=\left(6 \pi^{2} n_{\mathrm{F}}\right)^{1 / 3}$. The dimensionless quantity

$$
\lambda=\frac{16}{3}\left(k_{\mathrm{F}} a_{\mathrm{FB}}\right)^{2} \frac{n_{\mathrm{B}}}{n_{\mathrm{F}}}(1+\alpha)(1+1 / \alpha)
$$

measures the strength while

$$
k_{\mathrm{F}} \xi_{\mathrm{B}}=\frac{\sqrt{3 \pi}}{2} \sqrt{\frac{n_{F}}{n_{\mathrm{B}} k_{\mathrm{F}} a_{\mathrm{B}}}}
$$

characterizes the range of the mediated interaction. It is intuitively clear that increasing the strength and range of the pairing interaction will raise $T_{\mathrm{c}}$. The third dimensionless quantity is the ratio of the speed of sound in BEC $c_{\mathrm{B}}=\sqrt{g_{\mathrm{B}} n_{\mathrm{B}} / m_{\mathrm{B}}}$ and the Fermi velocity $v_{\mathrm{F}}=k_{\mathrm{F}} / m_{\mathrm{F}}$,

$$
\frac{c_{\mathrm{B}}}{v_{\mathrm{F}}}=\sqrt{\frac{2}{3 \pi}} \alpha \sqrt{\left(n_{\mathrm{B}} / n_{\mathrm{F}}\right)\left(k_{\mathrm{F}} a_{\mathrm{B}}\right)} .
$$

The larger this ratio is, the smaller the effects of retardation will be, and the higher the $T_{\mathrm{c}}$ will become.

A few comments are in order. First, Eqs. (13)-(15) show that when the mass ratio $\alpha$ increases, the interaction strength increases and its range is constant, while retardation effects decrease. This indicates that using a mixture of light bosons and heavy fermions favors a high $T_{\mathrm{c}}$, which we shall demonstrate explicitly below. Second, increasing $n_{\mathrm{B}} / n_{\mathrm{F}}$ will increase the speed of sound in the BEC and the interaction strength, but decrease its range. Likewise, increasing $k_{\mathrm{F}} a_{\mathrm{B}}$ will increase the $\mathrm{BEC}$ speed of sound but decrease the interaction range. The competition between these effects makes the dependence of $T_{\mathrm{c}}$ on $n_{\mathrm{B}} / n_{\mathrm{F}}$ and $k_{\mathrm{F}} a_{\mathrm{B}}$ a priori nontrivial. Finally, we cannot freely increase the scattering length $a_{\mathrm{FB}}$, as the system will phase separate (collapse) for sufficiently positive (negative) $a_{\mathrm{FB}}$. Within mean-field theory, the condition for avoiding such instabilities is [35]

$$
\left(k_{\mathrm{F}} a_{\mathrm{FB}}\right)^{2} \leq \frac{2 \pi}{(1+\alpha)(1+1 / \alpha)} k_{\mathrm{F}} a_{\mathrm{B}} .
$$

We emphasize however, that Eq. (16) most likely underestimates the region of stability for trapped Bose-Fermi mixtures, since it is based on mean-field theory and is derived for a homogeneous system ignoring finite size effects. Indeed, two recent experiments show that trapped Bose-Fermi mixtures are stable far beyond the condition given by Eq. (16), both for attractive [18] and repulsive interactions [19]. Phase separation for trapped Bose-Fermi mixtures was considered in Refs. [36-38].

Numerical results. - We now present numerical results for $T_{\mathrm{c}}$ for experimentally relevant Bose-Fermi mixtures. Since the BEC density is typically much higher than that of the fermions, we take $n_{\mathrm{B}} / n_{\mathrm{F}}=5$ for all the calculations.

Consider first the ${ }^{7} \mathrm{Li}^{1}{ }^{173} \mathrm{Yb}$ mixture, which has been experimentally realized [20]. It corresponds to a mass ratio $\alpha=173 / 7$ as high as currently possible with present atomic gas experiments, and we expect it to be the most favorable for achieving a high $T_{\mathrm{c}}$. We plot in Fig. 2 the critical temperature as a function of the fermionboson interaction strength $k_{\mathrm{F}} a_{\mathrm{FB}}$ obtained from the full Eliashberg theory using the boson-boson interaction 


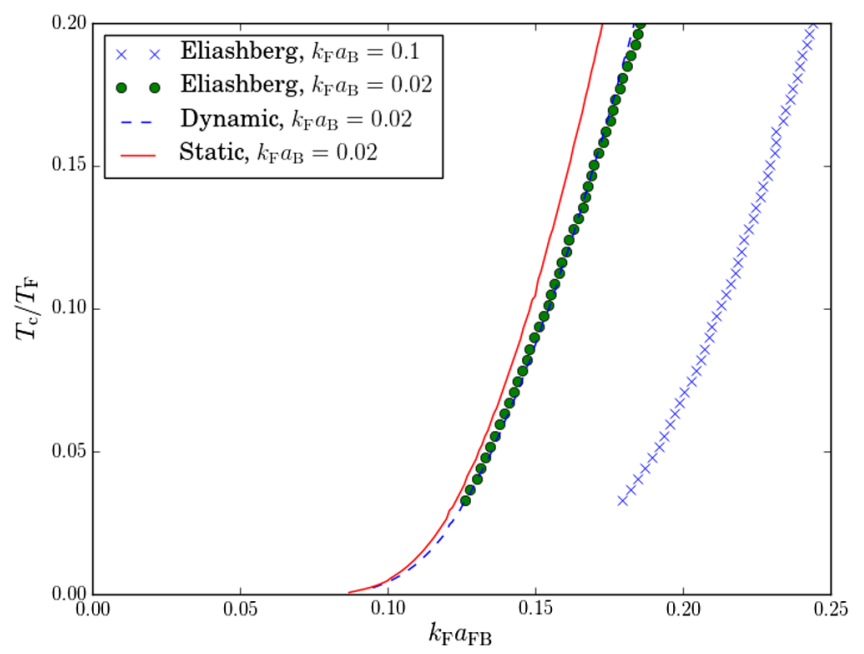

FIG. 2. Critical temperature for $p$-wave pairing in the ${ }^{7} \mathrm{Li}^{-173} \mathrm{Yb}$ mixture as a function of Fermi-Bose scattering length $k_{\mathrm{F}} a_{\mathrm{FB}}$. The dynamic and static labels refer to when the normal self-energy, and to when both the normal self-energy and retardation effects are ignored, respectively.

strengths $k_{\mathrm{F}} a_{\mathrm{B}}=0.1$ and $k_{\mathrm{F}} a_{\mathrm{B}}=0.02$. The critical temperature increases with $k_{\mathrm{F}} a_{\mathrm{FB}}$ as expected. Taking $T_{\mathrm{c}} / T_{\mathrm{F}}=$ 0.1 as a conservative estimate for what can be realized experimentally, this is reached at the relatively weak coupling strengths $k_{\mathrm{F}}\left|a_{\mathrm{FB}}\right| \simeq 0.155$ for $k_{\mathrm{F}} a_{\mathrm{B}}=0.02$ and $k_{\mathrm{F}}\left|a_{\mathrm{FB}}\right| \simeq 0.215$ for $k_{\mathrm{F}} a_{\mathrm{B}}=0.1$, where our theory is reliable. The critical temperature is higher for $k_{\mathrm{F}} a_{\mathrm{B}}=$ 0.02 compared to $k_{\mathrm{F}} a_{\mathrm{B}}=0.1$, showing that when $k_{\mathrm{F}} a_{\mathrm{B}}$ decreases, the increase in interaction range more than compensates for the increasing retardation effects.

For comparison, we also plot in Fig. 2 the critical temperature for $k_{\mathrm{F}} a_{\mathrm{B}}=0.02$ obtained when both retardation effects and the normal self-energy are neglected; i.e., we use the induced interaction evaluated at zero frequency and set $\Sigma(p)=0$. Such a static theory significantly overestimates $T_{\mathrm{c}}$, which for this particular mixture is mainly because it neglects retardation effects. This can be seen when we include retardation but still neglect the normal self-energy $\Sigma(p)$, the resulting $T_{\mathrm{c}}$ largely agrees with that obtained from the full theory, as shown in Fig. 2. Note that our results are independent of the sign of $a_{\mathrm{FB}}$, since the induced interaction is second order in $a_{\mathrm{FB}}$.

Equation (16) predicts that a homogeneous ${ }^{7} \mathrm{Li}^{173} \mathrm{Yb}$ mixture will collapse or phase separate for $k_{\mathrm{F}}\left|a_{\mathrm{FB}}\right| \gtrsim 0.069$ for $k_{\mathrm{F}} a_{\mathrm{B}}=0.02$ and $k_{\mathrm{F}}\left|a_{\mathrm{FB}}\right| \gtrsim 0.153$ for $k_{\mathrm{F}} a_{\mathrm{B}}=0.1$. Taking into account that trapped mixtures are stable well beyond these critical values, we conclude from Fig. 2 that a ${ }^{7} \mathrm{Li}^{173} \mathrm{Yb}$ mixture is a promising platform for realizing a $p$-wave superfluid caused by an induced interaction.

Consider next the two experimentally relevant mixtures ${ }^{23} \mathrm{Na}^{-}{ }^{40} \mathrm{~K}$ [15] and ${ }^{87} \mathrm{Rb}-{ }^{40} \mathrm{~K}$ [12-14], which have almost the inverse mass ratios. In Fig. 3, we plot $T_{\mathrm{c}}$ for $k_{\mathrm{F}} a_{\mathrm{B}}=0.02$ obtained using three theoretical approaches: (i) the full

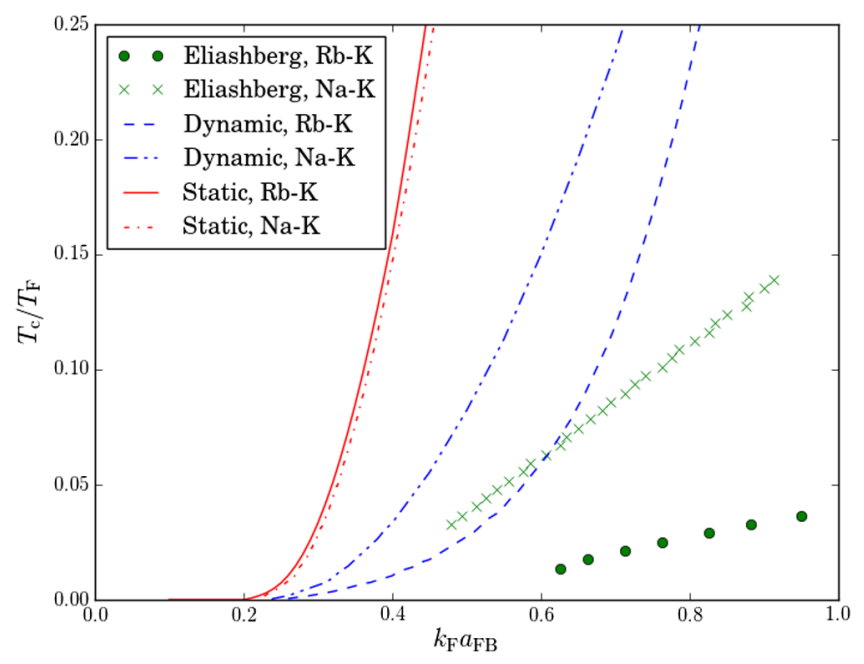

FIG. 3. Critical temperature for the ${ }^{23} \mathrm{Na}^{4}{ }^{40} \mathrm{~K}$ and ${ }^{87} \mathrm{Rb}-{ }^{40} \mathrm{~K}$ mixtures as a function of $k_{\mathrm{F}} a_{\mathrm{FB}}$ for $k_{\mathrm{F}} a_{\mathrm{B}}=0.02$. The static and dynamic labels refer to the same theories as in Fig. 2.

Eliashberg theory, (ii) including retardation but neglecting the normal self-energy, and (iii) neglecting both retardation by using the zero frequency induced interaction and the normal self-energy. The critical temperatures of the two mixtures are almost the same when both retardation and self-energy effects are ignored. This can be understood from Eq. (13), since the dimensionless interaction is nearly the same for the two mixtures. However, $T_{\mathrm{c}}$ is much higher for the ${ }^{23} \mathrm{Na}^{40} \mathrm{~K}$ mixture, when retardation effects are included. This is because retardation is less important for light bosons due to their higher speed of sound, see Eq. (15). Finally, Fig. 3 shows that the normal self-energy $\Sigma(p)$ also suppresses $T_{\mathrm{c}}$ most for the ${ }^{87} \mathrm{Rb}-{ }^{40} \mathrm{~K}$ mixture. The reason is that excitations in the BEC cost less energy for heavy bosons, which leads to larger self-energy effects. The fact that the ${ }^{23} \mathrm{Na}^{-}{ }^{40} \mathrm{~K}$ mixture has a much higher $T_{c}$ than the ${ }^{87} \mathrm{Rb}-{ }^{40} \mathrm{~K}$ mixture in the full Eliashberg theory nicely illustrates a main result of the present Letter: a mixture of light bosons and heavy fermions is more favorable to achieve a high $T_{\mathrm{c}}$. This is further corroborated by the fact that, according to Eq. (16), the two mixtures become unstable almost at the same coupling strength, $k_{\mathrm{F}}\left|a_{\mathrm{FB}}\right|=$ 0.171 for ${ }^{23} \mathrm{Na}^{40} \mathrm{~K}$ and $k_{\mathrm{F}}\left|a_{\mathrm{FB}}\right|=0.165$ for ${ }^{87} \mathrm{Rb}^{4}{ }^{40} \mathrm{~K}$.

In order to investigate the effects of the boson-boson interaction, we plot in Fig. 4 the critical temperature as a function of $k_{\mathrm{F}} a_{\mathrm{B}}$ for the ${ }^{7} \mathrm{Li}^{173} \mathrm{Yb},{ }^{23} \mathrm{Na}-{ }^{40} \mathrm{~K}$, and ${ }^{7} \mathrm{Li}-{ }^{6} \mathrm{Li}$ mixtures $[9,15,20]$. For all three mixtures, $T_{\mathrm{c}}$ decreases with $k_{\mathrm{F}} a_{\mathrm{B}}$. Thus, although the sound velocity of the BEC increases with $k_{\mathrm{F}} a_{\mathrm{B}}$ thereby reducing retardation effects, this effect is overwhelmed by the corresponding reduction in the interaction range, so that the net effect is a suppression of $T_{\mathrm{c}}$ with increasing $k_{\mathrm{F}} a_{\mathrm{B}}$. The suppression is largest for the ${ }^{7} \mathrm{Li}^{173} \mathrm{Yb}$ mixture, since retardation effects are already small for light bosons so that a decrease in the interaction range has a larger relative effect. 


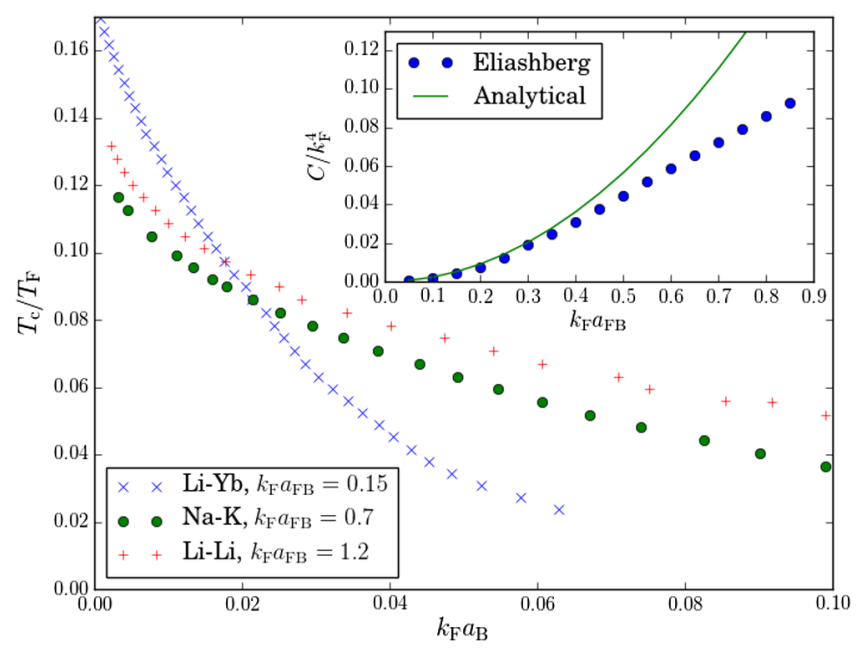

FIG. 4. Critical temperature as function of the Bose-Bose scattering length $k_{\mathrm{F}} a_{\mathrm{B}}$ for three mixtures. Inset shows the contact $C$ for the ${ }^{23} \mathrm{Na}^{-40} \mathrm{~K}$ mixture.

Finally, we plot in the inset of Fig. 4 the contact $C=\lim _{k \rightarrow \infty}\left\langle a_{\mathbf{k}}^{\dagger} a_{\mathbf{k}}\right\rangle k^{4}[39-41]$ for a ${ }^{23} \mathrm{Na}^{-}{ }^{40} \mathrm{~K}$ mixture with $k_{\mathrm{F}} a_{\mathrm{B}}=0.1$ and temperature $T=0.125 T_{\mathrm{F}}$. It increases with $k_{\mathrm{F}} a_{\mathrm{BF}}$ as expected. Since the Fock self-energy, Eq. (9) includes all dominant second order diagrams for a fermion interacting with a BEC $[30,42]$, our theory recovers the exact second order result [33]

$$
C=\frac{\left(2 k_{\mathrm{F}} a_{\mathrm{FB}}\right)^{2} n_{\mathrm{B}}}{9 \pi^{2} n_{\mathrm{F}}} k_{\mathrm{F}}^{4} .
$$

We see from the inset of Fig. 4 that the numerical results indeed approach Eq. (17) for $k_{\mathrm{F}} a_{\mathrm{FB}} \ll 1$ thereby illustrating the accuracy of our approach.

Experimental realization.-As mentioned in the introduction, a wide range of atomic Bose-Fermi mixtures have already been realized experimentally [9-20]. These experiments are inherently inhomogeneous due to a harmonic trap, and the observability of the superfluid state requires that the spatial size of the phase is sufficiently large compared to the coherence length of the Cooper pairs. Recent experiments show that trapped Bose-Fermi mixtures have a significant overlap region between the atomic species over a large range of interaction strengths $[18,19]$. This implies that the $p$-wave superfluid should occupy a sizeable region in the trap. For the detection of the superfluid state, one could observe the formation of vortices under rotation [43], measure the reduction of the moment of inertia [44], or directly probe the pairing gap by local Bragg spectroscopy [45].

Conclusions.-We presented a strong coupling theory for the $p$-wave pairing of spin polarized fermions in a BEC, which takes into account the full frequency and momentum dependence of the induced interaction between the fermions caused by the exchange of phonons in the BEC.
Focusing on experimentally relevant systems, we calculated the critical temperature varying the boson-boson and boson-fermion interaction strengths, as well as their mass ratio. Both retardation as well as self-energy effects were shown to significantly affect $T_{\mathrm{c}}$. Our systematic analysis allowed us to identity the most suitable system for which the $p$-wave superfluidity can be achieved. In particular, we showed that it is within experimental reach using a mixture of light bosons and heavy fermions. The $p$-wave superfluid can be considered as the many-body limit of a gas of bi-polarons [46], where the size of the bi-polarons is much larger than their average distance. This opens up the intriguing possibility to study the BEC-BCS crossover in an entirely new setting by varying the fermion density.

We thank R. Grimm, M. Zaccanti, and J. Thywissen for useful discussions. G. M. B. wishes to acknowledge the support of the Villum Foundation and the Danish Council of Independent Research. Z. W. acknowledges the support by the Shenzhen Science and Technology Innovation Commission (Grants No. ZDSYS20170303165926217, No. JCYJ20170412152620376) and Guangdong Innovative and Entrepreneurial Research Team Program (Grant No. 2016ZT06D348). The computational time was provided by the Triton cluster of the Aalto School of Science.

*jami.kinnunen@aalto.fi

†wuzg@sustc.edu.cn

tbruungmb@phys.au.dk

[1] J. Schrieffer, Theory of Superconductivity, Advanced Book Program Series (Avalon Publishing, New York, 1983).

[2] D. Vollhardt and P. Wölfle, The Superfluid Phases of Helium 3 (Taylor and Francis, London, 1990).

[3] J. G. Bednorz and K. A. Müller, Z. Phys. B 64, 189 (1986).

[4] Y. Kamihara, T. Watanabe, M. Hirano, and H. Hosono, J. Am. Chem. Soc. 130, 3296 (2008).

[5] C. A. Regal, M. Greiner, and D. S. Jin, Phys. Rev. Lett. 92, 040403 (2004).

[6] M. W. Zwierlein, C. A. Stan, C. H. Schunck, S. M. F. Raupach, A. J. Kerman, and W. Ketterle, Phys. Rev. Lett. 92, 120403 (2004).

[7] H. Heiselberg, C. J. Pethick, H. Smith, and L. Viverit, Phys. Rev. Lett. 85, 2418 (2000).

[8] M. J. Bijlsma, B. A. Heringa, and H. T. C. Stoof, Phys. Rev. A 61, 053601 (2000).

[9] I. Ferrier-Barbut, M. Delehaye, S. Laurent, A. T. Grier, M. Pierce, B. S. Rem, F. Chevy, and C. Salomon, Science 345, 1035 (2014).

[10] R. Roy, A. Green, R. Bowler, and S. Gupta, Phys. Rev. Lett. 118, 055301 (2017).

[11] X.-C. Yao, H.-Z. Chen, Y.-P. Wu, X.-P. Liu, X.-Q. Wang, X. Jiang, Y. Deng, Y.-A. Chen, and J.-W. Pan, Phys. Rev. Lett. 117, 145301 (2016).

[12] M. Zaccanti, C. D’Errico, F. Ferlaino, G. Roati, M. Inguscio, and G. Modugno, Phys. Rev. A 74, 041605 (2006). 
[13] S. Ospelkaus, C. Ospelkaus, L. Humbert, K. Sengstock, and K. Bongs, Phys. Rev. Lett. 97, 120403 (2006).

[14] M. Taglieber, A.-C. Voigt, T. Aoki, T. W. Hänsch, and K. Dieckmann, Phys. Rev. Lett. 100, 010401 (2008).

[15] J. W. Park, C.-H. Wu, I. Santiago, T. G. Tiecke, S. Will, P. Ahmadi, and M. W. Zwierlein, Phys. Rev. A 85, 051602 (2012).

[16] M.-S. Heo, T. T. Wang, C. A. Christensen, T. M. Rvachov, D. A. Cotta, J.-H. Choi, Y.-R. Lee, and W. Ketterle, Phys. Rev. A 86, 021602 (2012).

[17] V. D. Vaidya, J. Tiamsuphat, S. L. Rolston, and J. V. Porto, Phys. Rev. A 92, 043604 (2015).

[18] B. J. DeSalvo, K. Patel, J. Johansen, and C. Chin, Phys. Rev. Lett. 119, 233401 (2017).

[19] R. S. Lous, I. Fritsche, M. Jag, F. Lehmann, E. Kirilov, B. Huang, and R. Grimm, Phys. Rev. Lett. 120, 243403 (2018).

[20] F. Schäfer, N. Mizukami, P. Yu, S. Koibuchi, A. Bouscal, and Y. Takahashi, Phys. Rev. A 98, 051602 (2018).

[21] D. V. Efremov and L. Viverit, Phys. Rev. B 65, 134519 (2002).

[22] F. Matera, Phys. Rev. A 68, 043624 (2003).

[23] A. Bulgac, M. M. Forbes, and A. Schwenk, Phys. Rev. Lett. 97, 020402 (2006).

[24] K. Suzuki, T. Miyakawa, and T. Suzuki, Phys. Rev. A 77, 043629 (2008).

[25] A. Bulgac and S. Yoon, Phys. Rev. A 79, 053625 (2009).

[26] F. Matera and A. Dellafiore, Eur. Phys. J. D 65, 515 (2011).

[27] Y.-J. Wu, J. He, C.-L. Zang, and S.-P. Kou, Phys. Rev. B 86, 085128 (2012).

[28] J. Okamoto, L. Mathey, and W.-M. Huang, Phys. Rev. A 95, 053633 (2017).

[29] S. P. Rath and R. Schmidt, Phys. Rev. A 88, 053632 (2013).
[30] R. S. Christensen, J. Levinsen, and G. M. Bruun, Phys. Rev. Lett. 115, 160401 (2015).

[31] J. Bardeen, G. Baym, and D. Pines, Phys. Rev. Lett. 17, 372 (1966).

[32] G. Mahan, Many-Particle Physics (Kluwer Academic/ Plenum Publishers, New York, 2000).

[33] See Supplemental Material at http://link.aps.org/ supplemental/10.1103/PhysRevLett.121.253402 for the derivation of the Eliashberg equations and details of the numerical procedure.

[34] J. J. Kinnunen and G. M. Bruun, Phys. Rev. A 91, 041605 (2015).

[35] L. Viverit, C. J. Pethick, and H. Smith, Phys. Rev. A 61, 053605 (2000).

[36] K. Mølmer, Phys. Rev. Lett. 80, 1804 (1998).

[37] N. Nygaard and K. Mølmer, Phys. Rev. A 59, 2974 (1999).

[38] R. Roth, Phys. Rev. A 66, 013614 (2002).

[39] S. Tan, Ann. Phys. (Amsterdam) 323, 2952 (2008).

[40] S. Tan, Ann. Phys. (Amsterdam) 323, 2971 (2008).

[41] E. Braaten, in The BCS-BEC Crossover and the Unitary Fermi Gas, edited by W. Zwerger (Springer, New York, 2012).

[42] A. Camacho-Guardian and G. M. Bruun, Phys. Rev. X 8, 031042 (2018).

[43] M. W. Zwierlein, J. R. Abo-Shaeer, A. Schirotzek, C. H. Schunck, and W. Ketterle, Nature (London) 435, 1047 (2005).

[44] S. Riedl, E. R. S. Guajardo, C. Kohstall, J. H. Denschlag, and R. Grimm, New J. Phys. 13, 035003 (2011).

[45] S. Hoinka, P. Dyke, M. G. Lingham, J. J. Kinnunen, G. M. Bruun, and C. J. Vale, Nat. Phys. 13, 943 (2017).

[46] A. Camacho-Guardian, L. A. Peña Ardila, T. Pohl, and G. M. Bruun, Phys. Rev. Lett. 121, 013401 (2018). 